\title{
Surveillance of Respiratory Viruses in Long Term Care Facilities
}

\author{
Mary M. Checovich ${ }^{1}$, Shari Barlow ${ }^{1}$, Peter Shult ${ }^{2}$, Erik Reisdorf ${ }^{2}$, Jonathan L. Temte ${ }^{1}$ \\ ${ }^{1}$ Family Medicine \& Community Health, University of Wisconsin-Madison, Madison, Wisconsin, United States, ${ }^{2}$ Wisconsin State Laboratory of \\ Hygiene, Madison, Wisconsin, United States
}

\section{Objective}

To assess the feasibility of conducting respiratory virus surveillance for residents of long term care facilities (LTCF) using simple nasal swab specimens and to describe the virology of acute respiratory infections (ARI) in LCTFs.

\section{Introduction}

Although residents of LTCFs have high morbidity and mortality associated with ARIs, there is very limited information on the virology of ARI in LTCFs [1,2]. Moreover, most virological testing of LCTF residents is reactive and is triggered by a resident meeting selected surveillance criteria. We report on incidental findings from a prospective trial of introducing rapid influenza diagnostic testing (RIDT) in ten Wisconsin LTCFs over a two-year period with an approach of testing any resident with ARI.

\section{Methods}

Any resident with new onset of respiratory symptoms consistent with ARI had a nasal swab specimen collected for RIDT by nursing staff. Following processing for RIDT (Quidel Sofia Influenza A+B FIA), the residual swab was placed into viral transport medium and forwarded to the Wisconsin State Laboratory of Hygiene and tested for influenza using RT-PCR (IVD CDC Human Influenza Virus Real-Time RT-PCR Diagnostic Panel), and for 17 viruses (Luminex NxTAG Respiratory Pathogen Panel [RPP]). The numbers of viruses in each of 7 categories [influenza A (FluA), influenza B (FluB), coronaviruses (COR), human metapneumovirus (hMPV), parainfluenza (PARA), respiratory syncytial virus (RSV) and rhinovirus/enterovirus (R/E)], across the two years were compared using chi-square.

\section{Results}

Totals of 164 and 190 specimens were submitted during 2016-2017 and 2017-2018, respectively. RPP identified viruses in 56.2\% of specimens, with no difference in capture rate between years (55.5\% vs. 56.8\%). Influenza A (21.5\%), influenza B (16.5\%), RSV $(19.0 \%)$ and hMPV (16.5\%) accounted for $73.5 \%$ of all detections, while coronaviruses (15.5\%), rhino/enteroviruses (8.5\%) and parainfluenza $(2.5 \%)$ were less common. Specific distribution of viruses varied significantly across the two years $\left(\right.$ Table: $X^{2}=48.1$, $\mathrm{df}=6 ; \mathrm{p}<0.001)$.

\section{Conclusions}

Surveillance in LTCFs using nasal swabs collected for RIDT is highly feasible and yields virus identification rates similar to those obtained in clinical surveillance of ARI with collection of nasopharyngeal specimens by clinicians and those obtained in a schoolbased surveillance project of ARI with collection of combined nasal and oropharyngeal specimens collected by trained research assistants. Significant differences in virus composition occurred across the two study years. RSV varied little between years while hMPV demonstrated wide variation. Simple approaches to surveillance may provide a more comprehensive assessment of respiratory viruses in LTCF settings.

\section{Acknowledgement}

Funding provided by the Wisconsin Partnership Program / Partnership Education and Research Committee. In-kind support was provided by Quidel Corporation. We appreciate contributions from our LTCF study sites.

\section{References}

1. Uršič T, Gorišek Miksić N, Lusa L, Strle F, Petrovec M. 2016. Viral respiratory infections in a nursing home: a six-month prospective study. BMC Infect Dis. 16, 637. Published online Nov 2016. doi:https://doi.org/10.1186/s12879-016-1962-8. PubMed 


\section{OJPHI}

ISDS 2019 Conference Abstracts

2. Masse S, Capai L, Falchi A. 2017. Epidemiology of Respiratory Pathogens among Elderly Nursing Home Residents with Acute Respiratory Infections in Corsica, France, 2013-2017. BioMed Res Int. 2017, 1. Published online Dec 2017. doi:https://doi.org/10.1155/2017/1423718. PubMed

Distribution of respiratory viruses in LTCFs over two sequential seasons.

\begin{tabular}{|c|c|c|c|c|c|c|c|}
\hline \multirow[t]{2}{*}{ Respiratory Virus Season } & \multicolumn{7}{|c|}{ Virus Category (percent of specimens) } \\
\hline & FluA & FluB & COR & $\begin{array}{c}\mathbf{h M P} \\
\mathbf{V}\end{array}$ & $\begin{array}{c}\text { PAR } \\
\text { A }\end{array}$ & $\mathbf{R S V}$ & $\mathbf{R} / \mathbf{E}$ \\
\hline $2017 / 2018$ & 17.6 & 24.2 & 20.9 & 0 & 5.5 & 18.7 & 13.2 \\
\hline $2017 / 2018$ & 24.8 & 10.1 & 11.0 & 30.3 & 0 & 19.3 & 4.6 \\
\hline Combined & 21.5 & 16.5 & 15.5 & 16.5 & 2.5 & 19.0 & 8.5 \\
\hline
\end{tabular}

